\title{
Synchronization and Antisynchronization of a Planar Oscillation of Satellite in an Elliptic Orbit via Active Control
}

\author{
Mohammad Shahzad \\ Department of Information Technology, Higher College of Technology, Muscat, Oman \\ Correspondence should be addressed to Mohammad Shahzad, dmsinfinite@gmail.com
}

Received 6 April 2011; Revised 4 June 2011; Accepted 22 June 2011

Academic Editor: Derong Liu

Copyright (๑) 2011 Mohammad Shahzad. This is an open access article distributed under the Creative Commons Attribution License, which permits unrestricted use, distribution, and reproduction in any medium, provided the original work is properly cited.

\begin{abstract}
We have investigated the synchronization and antisynchronization behaviour of two identical planar oscillation of a satellite in elliptic orbit evolving from different initial conditions using the active control technique based on the Lyapunov stability theory and the Routh-Hurwitz criteria. The designed controller, with our own choice of the coefficient matrix of the error dynamics that satisfy the Lyapunov stability theory and the Routh-Hurwitz criteria, is found to be effective in the stabilization of the error states at the origin, thereby, achieving synchronization and antisynchronization between the states variables of two nonlinear dynamical systems under consideration. The results are validated by numerical simulations using mathematica.
\end{abstract}

\section{Introduction}

In the last two decades, considerable research has been done in nonlinear dynamical systems and their various properties. One of the most important property of nonlinear dynamical systems is synchronization, which classically represents the entrainment of frequencies of oscillators due to weak interactions [1-3]. Studies in this field are partly motivated by experimental realization in lasers, electronic circuits, plasma discharge, and chemical reactions [2-4]. Synchronization techniques have been improved in recent years, and many different methods are applied theoretically as well as experimentally to synchronize the chaotic systems. Some of them are nonlinear feedback method [5], adaptive control method [6], antisynchronization method [7], sliding mode control method [8], and chaos synchronization using active control, which is introduced in [9] is one of these methods. Notable among these methods, chaos synchronization using active control scheme has recently been widely accepted as an efficient technique to synchronize the chaotic systems. The reason is because it can be used to synchronize identical as well as nonidentical systems; a feature that gives it an advantage over other synchronizing methods. This method based on the Lyapunov stability theory and the Routh-Hurwitz criteria to active control in order to achieve stable synchronization has been applied to many practical systems such as the electronic circuits, which model a third-order "jerk" equation [10], Lorenz, Chen, and Lü system [11], geophysical systems [12], nonlinear equations of acoustic gravity waves (LorenzStenflo system) [13], Van-der Pol-Duffing oscillator [14], forced damped pendulum [15], nuclear magnetic resonance modeled by the Bloch equations [16], parametrically excited oscillators [17], permanent magnet reluctance machine [18], inertial ratchets [19], RCL-shunted Josephson junction [20], and modified projective synchronization [21].

In this article, we have applied the active control technique based on the Lyapunov stability theory and the RouthHurwitz criteria to study the synchronization and antisynchronization behavior of two identical planar oscillation of a satellite in elliptic orbit evolving from different initial conditions. The system under consideration is chaotic for some values of parameter involved in the system. In synchronization, the two systems (master and slave) are synchronized and start with different initial conditions. The same problem may be treated as the design of control laws for full chaotic slave system using the known information of the master system so as to ensure that the controlled receiver synchronizes with the master system. Hence, the slave chaotic system completely traces the dynamics of the master system in the course of time. The aim of this study is to trace the chaotic 
dynamics of the planar oscillation of a satellite in elliptic orbit based on synchronization and antisynchronization. To the best of my knowledge nobody studied this before.

\section{Description of the Model}

Elliptically orbiting planar oscillations of satellites in the solar system make an interesting study, and significant contributions to this end can be found [22-30], all of which have studied the influence of certain perturbative forces, such as solar radiation pressure, tidal force, and air resistance. In the present work, we consider the planar oscillation of a satellite in elliptic orbit with the spin axis fixed perpendicular to the orbital plane. Let the long axis of the satellite makes an angle $\theta$ with a reference axis that is fixed in inertial space, the long axis of the satellite makes an angle $\psi$ with satellites planet centre line [31], and the satellite to be a triaxial ellipsoid with principal moments of inertia $A<B<C$, where $C$ is the moment about the spin axis. The orbit is taken to be a fixed ellipse with semimajor axis $a$, eccentricity $e$, true anomaly $f$, and instantaneous radius $r$. The equation of motion of satellite planar oscillation in an elliptic orbit around the earth is

$$
2 C \ddot{\theta}-3(B-A) \frac{G m_{p}}{r^{3}} \sin 2 \psi=0 .
$$

Taking $3(B-A) / C=\omega_{0}^{2}, G m_{p}=\mu, \psi=f-\theta$ and $l / r=$ $1+e \cos f$, (1) can be written as

$$
\frac{d^{2} \theta}{d t^{2}}=\frac{\mu \omega_{0}^{2}}{l^{3}}(1+e \cos f)^{3} \sin 2(f-\theta) .
$$

In order to reduce three variables $\psi, \theta$, and $f$ we are taking $f$ as independent variable.

Now,

$$
\begin{aligned}
& \frac{d \theta}{d t}=\frac{d \theta}{d f} \frac{d f}{d t}=\dot{f} \frac{d \theta}{d f}=\frac{h}{r^{2}} \frac{d \theta}{d f}, \quad\left(\text { using } r^{2} \dot{f}=h\right), \\
& \frac{d^{2} \theta}{d t^{2}}=\frac{-2 h \dot{r}}{r^{3}} \frac{d \theta}{d f}+\frac{h}{r^{2}} \frac{d^{2} \theta}{d f^{2}} \frac{d f}{d t}, \\
& \frac{d^{2} \theta}{d t^{2}}=\frac{-2 h \dot{r}}{r^{3}} \frac{d \theta}{d f}+\frac{h^{2}}{r^{4}} \frac{d^{2} \theta}{d f^{2}} \quad\left(\text { using } r^{2} \dot{f}=h\right) .
\end{aligned}
$$

Now, from $l / r=1+e \cos f$, we have

$$
\begin{aligned}
& \dot{r}=\frac{-l(-e \sin f) \dot{f}}{(1+e \cos f)^{2}}, \\
& \dot{r}=\frac{e l \sin f}{(1+e \cos f)^{2}} \frac{h}{r^{2}} .
\end{aligned}
$$

Using (3) and (4) in (2), we have

$$
\begin{gathered}
\frac{h^{2}}{r^{4}} \frac{d^{2} \theta}{d f^{2}}-\frac{2 e l h^{2} \sin f}{r^{5}(1+e \cos f)^{2}} \frac{d \theta}{d f}=\frac{\mu \omega_{0}^{2}}{r^{3}} \sin 2(f-\theta) \\
\frac{h^{2}}{r^{4}} \frac{d^{2} \theta}{d f^{2}}-\frac{2 e h^{2} \sin f}{r^{4}(1+e \cos f)} \frac{d \theta}{d f} \\
=\frac{h^{2} \omega_{0}^{2}}{l r^{3}} \sin 2(f-\theta) \quad\left(\text { using } h^{2}=\mu l\right), \\
\frac{d^{2} \theta}{d f^{2}}=\frac{2 e \sin f}{(1+e \cos f)} \frac{d \theta}{d f}+\omega_{0}^{2} \frac{\sin 2(f-\theta)}{(1+e \cos f)} .
\end{gathered}
$$

Using the binomial expansion for $(1+e \cos f)^{-1}$ and for very small value of $e$ ignoring the higher order terms in $e$, we have

$$
\frac{d^{2} \theta}{d f^{2}}=2 e \sin f \frac{d \theta}{d f}+\frac{\omega_{0}^{2}}{2}(1-e \cos f) \sin 2(f-\theta) .
$$

\section{Synchronization via Active Control}

For a system of two coupled chaotic oscillators, the master system $(\dot{x}=f(x, y))$ and the slave system $(\dot{y}=g(x, y))$, where $x(t)$ and $y(t)$ are the phase space (state variables), and $f(x, y)$ and $g(x, y)$ are the corresponding nonlinear functions, synchronization in a direct sense implies $\lim _{t \rightarrow \infty}|x(t)-y(t)| \rightarrow 0$. When this occurs the coupled systems are said to be completely synchronized. Chaos synchronization is related to the observer problem in control theory [32]. The problem may be treated as the design of control laws for full chaotic slave system using the known information of the master system so as to ensure that the controlled receiver synchronizes with the master system. Hence, the slave chaotic system completely traces the dynamics of the master in the course of time.

In order to formulate the active controllers, we write the system (6) in two first-order differential equations as shown below.

Let $d \theta / d f=x_{1}^{\prime}$ and $d^{2} \theta / d f^{2}=x_{2}^{\prime}$, then we have

$$
\begin{gathered}
x_{1}^{\prime}=x_{2}, \\
x_{2}^{\prime}=2 e x_{1}^{\prime} \sin f+\frac{\omega_{0}^{2}}{2}(1-e \cos f) \sin 2\left(f-x_{1}\right) .
\end{gathered}
$$

Let us define another system

$$
y_{1}^{\prime}=y_{2}+u_{1}(f),
$$

$$
y_{2}^{\prime}=2 e y_{1}^{\prime} \sin f+\frac{\omega_{0}^{2}}{2}(1-e \cos f) \sin 2\left(f-y_{1}\right)+u_{2}(f),
$$

where (7) and (8) are called the master system and the slave system, respectively, and in slave system, $u_{1}(f)$ and $u_{2}(f)$ are control functions to be determined. Let $e_{1}(f)=y_{1}(f)-$ $x_{1}(f)$ and $e_{2}(f)=y_{2}(f)-x_{2}(f)$ be the synchronization 
errors such that $\lim _{f \rightarrow \infty} e_{i}(f) \rightarrow 0$ for $i=1,2$. From (7) and (8), we have

$$
\begin{aligned}
e_{1}^{\prime}(f)= & e_{2}(f)+u_{1}(f), \\
e_{2}^{\prime}(f)= & 2 e e_{1}^{\prime}(f) \sin f+\frac{\omega_{0}^{2}}{2}(1-e \cos f) \\
& \times\left[\sin 2\left(f-y_{1}\right)-\sin 2\left(f-x_{1}\right)\right]+u_{2}(f) .
\end{aligned}
$$

In order to express (9) as only linear terms in $e_{1}(f)$ and $e_{2}(f)$, we redefine the control functions as follows:

$$
\begin{aligned}
u_{1}(f)= & v_{1}(f), \\
u_{2}(f)= & -2 e e_{1}^{\prime}(f) \sin f-\frac{\omega_{0}^{2}}{2}(1-e \cos f) \\
& \times\left[\sin 2\left(f-y_{1}\right)-\sin 2\left(f-x_{1}\right)\right]+v_{2}(f) .
\end{aligned}
$$

From (9) and (10), we have

$$
\begin{aligned}
& e_{1}^{\prime}(f)=e_{2}(f)+v_{1}(f), \\
& e_{2}^{\prime}(f)=v_{2}(f) .
\end{aligned}
$$

Equation (11) is the error dynamics, which can be interpreted as a control problem where the system to be controlled is a linear system with control inputs $v_{1}(f)=$ $v_{1}\left(e_{1}(f), e_{2}(f)\right)$ and $v_{2}(f)=v_{2}\left(e_{1}(f), e_{2}(f)\right)$. As long as these feedbacks stabilize the system, $\lim _{f \rightarrow \infty} e_{i}(f) \rightarrow 0$ for $i=1,2$. This simply implies that the two systems (7) and (8) evolving from different initial conditions are synchronized. As functions of $e_{1}(f)$ and $e_{2}(f)$, we choose $v_{1}(f)$ and $v_{2}(f)$ as follows:

$$
\left(\begin{array}{l}
v_{1}(f) \\
v_{2}(f)
\end{array}\right)=D\left(\begin{array}{l}
e_{1}(t) \\
e_{2}(t)
\end{array}\right),
$$

where $D=\left(\begin{array}{ll}a & b \\ c & d\end{array}\right)$ is a $2 \times 2$ constant feedback matrix to be determined. Hence the error system (11) can be written as

$$
\left(\begin{array}{l}
e_{1}^{\prime}(f) \\
e_{2}^{\prime}(f)
\end{array}\right)=C\left(\begin{array}{c}
e_{1}(f) \\
e_{2}(f)
\end{array}\right),
$$

where $C=\left(\begin{array}{cc}a & 1+b \\ c & d\end{array}\right)$ is the coefficient matrix.

According to the Lyapunov stability theory and the Routh-Hurwitz criteria, if

$$
\begin{gathered}
a+d<0, \\
c(1+b)-a d<0,
\end{gathered}
$$

then the eigen values of the coefficient matrix of error system (11) must be real or complex with negative real parts and, hence, stable synchronized dynamics between systems (7) and ( 8 ) is guaranteed. Let

$$
a+d=c(1+b)-a d=-E,
$$

where $E>0$ is a real number which is usually set equal to 1 . There are several ways of choosing the constant elements $a$, $b, c, d$ of matrix $D$ in order to satisfy the Lyapunov stability theory and the Routh-Hurwitz criteria (14).

\section{Numerical Simulation for Synchronization}

For the constant elements of feedback matrix, choosing $a=$ $d=-0.5$ and for the parameters involved in system under investigation, $e=0.15, \omega_{0}=0.3$ with the initial conditions $\left[x_{1}(0), y_{1}(0)\right]=[0,0]$, and $\left[x_{2}(0), y_{2}(0)\right]=[1,1.5]$, we have simulated the system under consideration using mathematica. The results obtained show that the system under consideration achieved synchronization. Phase portraits, time series analysis, and error diagrams are the witness of achieving synchronization between master and slave system. Further, it also has been confirmed by the convergence of the synchronization quality defined by

$$
e(f)=\sqrt{e_{1}^{2}(f)+e_{2}^{2}(f)} .
$$

Figures 1-6 confirms the convergence of the synchronization quality defined by (16).

\section{Antisynchronization via Active Control}

Antisynchronization of two coupled systems $\dot{x}=f(x, y)$ (master system) and $\dot{y}=g(x, y)$ (slave system) means $\lim _{t \rightarrow \infty}|x(t)+y(t)| \rightarrow 0$. This phenomenon has been investigated both experimentally and theoretically in many physical systems $[18,19,32-37]$. A recent study of the antisynchronization phenomenon in nonequilibrium systems suggests that it could be used as a technique for particle separation in a mixture of interacting particles [19].

In order to formulate the active controllers for Antisynchronization, we need to redefine the error functions as $e_{1}(f)=y_{1}(f)+x_{1}(f)$ and $e_{2}(f)=y_{2}(f)+x_{2}(f)$, where $e_{1}(f)$ and $e_{2}(f)$ are called the antisynchronization errors such that $\lim _{f \rightarrow \infty} e_{i}(f)(i=1,2) \rightarrow 0$. From (7) and (8), error dynamics can be written as

$$
\begin{aligned}
e_{1}^{\prime}(f)= & e_{2}(f)+u_{1}(f), \\
e_{2}^{\prime}(f)= & 2 e e_{1}^{\prime}(f) \sin f+\frac{\omega_{0}^{2}}{2}(1-e \cos f) \\
& \times\left[\sin 2\left(f-y_{1}\right)+\sin 2\left(f-x_{1}\right)\right]+u_{2}(f) .
\end{aligned}
$$

In order to express (17) as only linear terms in $e_{1}(f)$ and $e_{2}(f)$, we redefine the control functions as follows:

$$
\begin{aligned}
u_{1}(f)= & v_{1}(f), \\
u_{2}(f)= & -2 e e_{1}^{\prime}(f) \sin f-\frac{\omega_{0}^{2}}{2}(1-e \cos f) \\
& \times\left[\sin 2\left(f-y_{1}\right)+\sin 2\left(f-x_{1}\right)\right]+v_{2}(f) .
\end{aligned}
$$

From (17) and (18), we have

$$
\begin{aligned}
& e_{1}^{\prime}(f)=e_{2}(f)+v_{1}(f), \\
& e_{2}^{\prime}(f)=v_{2}(f) .
\end{aligned}
$$




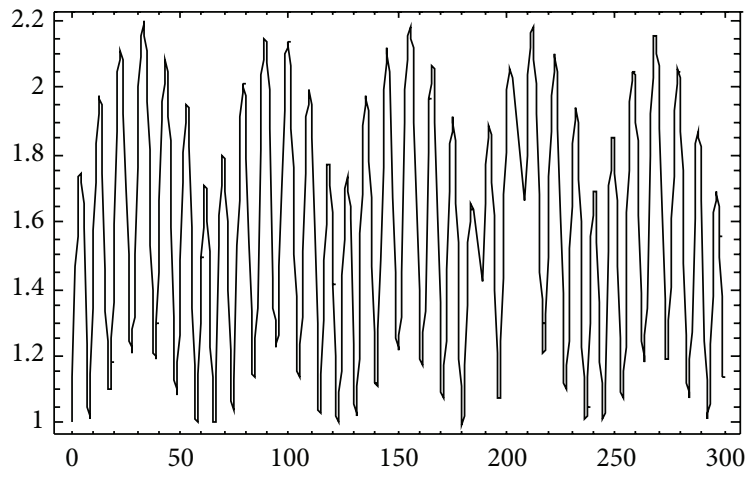

Figure 1: Phase plot of master system.

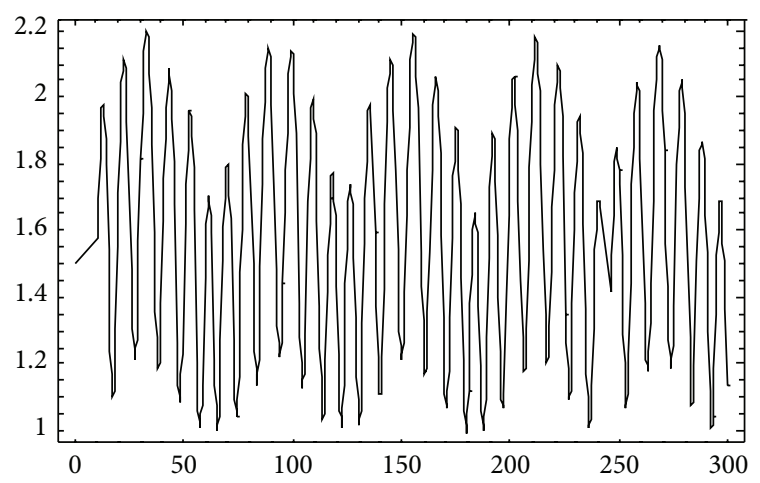

FIgUre 2: Phase plot of slave system.

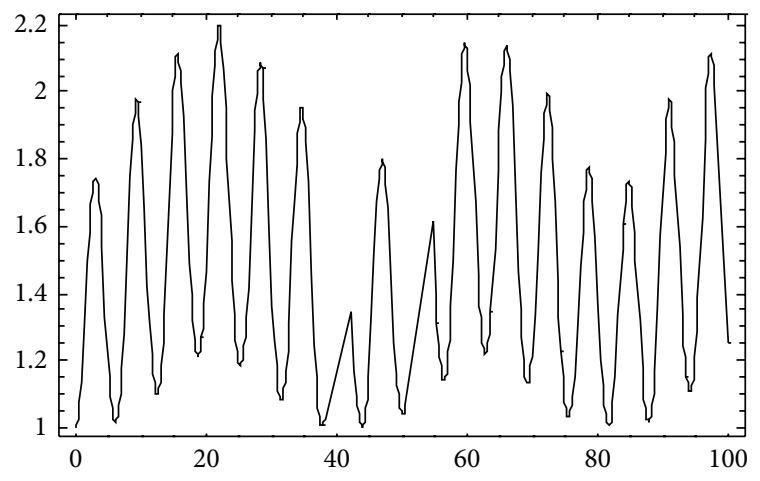

FIgURE 3: Time series analysis of master system.

Equation (19) is the error dynamics, which can be interpreted as a control problem where the system to be controlled is a linear system with control inputs $v_{1}(f)=v_{1}\left(e_{1}(f), e_{2}(f)\right.$ ), and $v_{2}(f)=v_{2}\left(e_{1}(f), e_{2}(f)\right)$. As long as these feedbacks stabilize the system, $\lim _{f \rightarrow \infty} e_{i}(f) \rightarrow 0$ for $i=1,2$. This simply implies that the two systems (7) and (8) evolving from different initial conditions are synchronized. As functions of $e_{1}(f)$ and $e_{2}(f)$, we choose $v_{1}(f)$ and $v_{2}(f)$ as follows:

$$
\left(\begin{array}{c}
v_{1}(f) \\
v_{2}(f)
\end{array}\right)=D\left(\begin{array}{c}
e_{1}(f) \\
e_{2}(f)
\end{array}\right),
$$

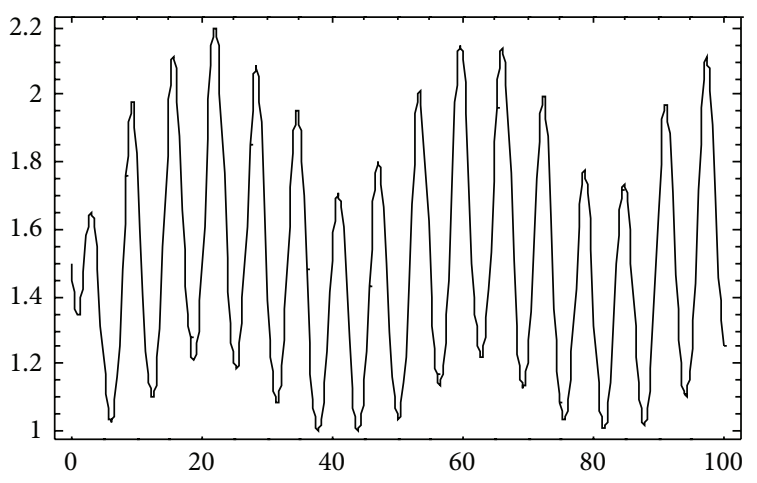

FIgURE 4: Time series analysis of slave system.

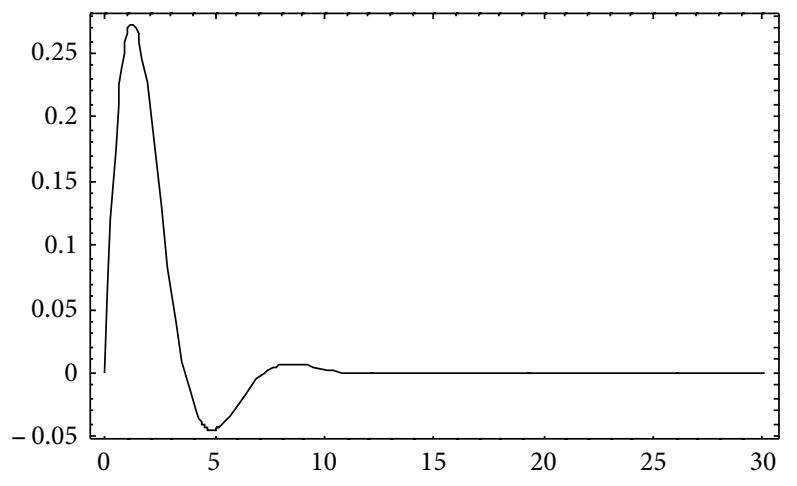

FIgURe 5: Time series analysis of $e_{1}(f)$.

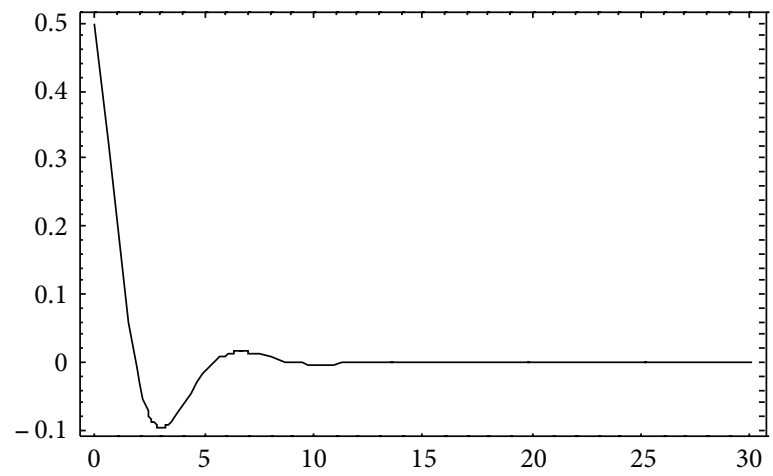

Figure 6: Time series analysis of $e_{2}(f)$.

where $D=\left(\begin{array}{ll}a & b \\ c & d\end{array}\right)$ is a $2 \times 2$ constant feedback matrix to be determined. Hence the error system (19) can be written as

$$
\left(\begin{array}{c}
e_{1}^{\prime}(f) \\
e_{2}^{\prime}(f)
\end{array}\right)=C\left(\begin{array}{c}
e_{1}(f) \\
e_{2}(f)
\end{array}\right),
$$

where $C=\left(\begin{array}{cc}a & 1+b \\ c & d\end{array}\right)$, is the coefficient matrix.

According to the Lyapunov stability theory and the RouthHurwitz criteria, if

$$
\begin{gathered}
a+d<0, \\
c(1+b)-a d<0,
\end{gathered}
$$




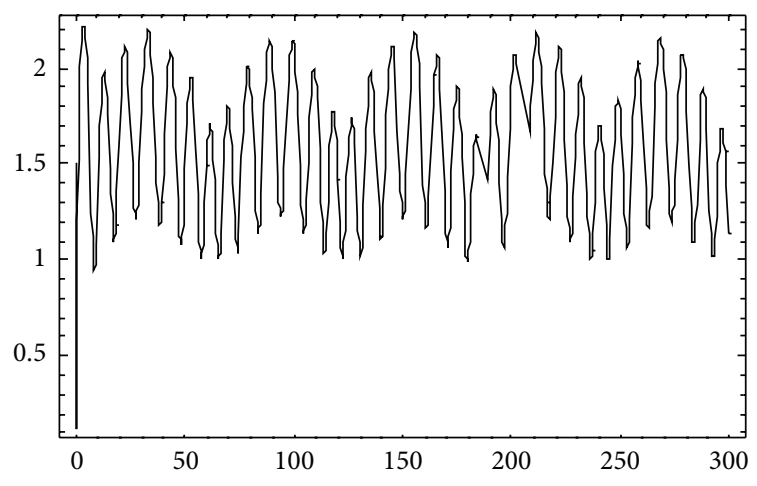

Figure 7: Phase plot of slave system.

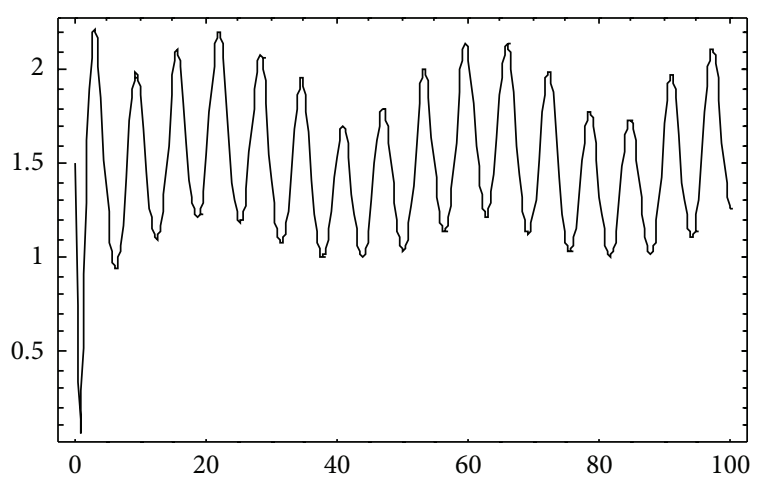

Figure 8: Time series analysis of slave system.

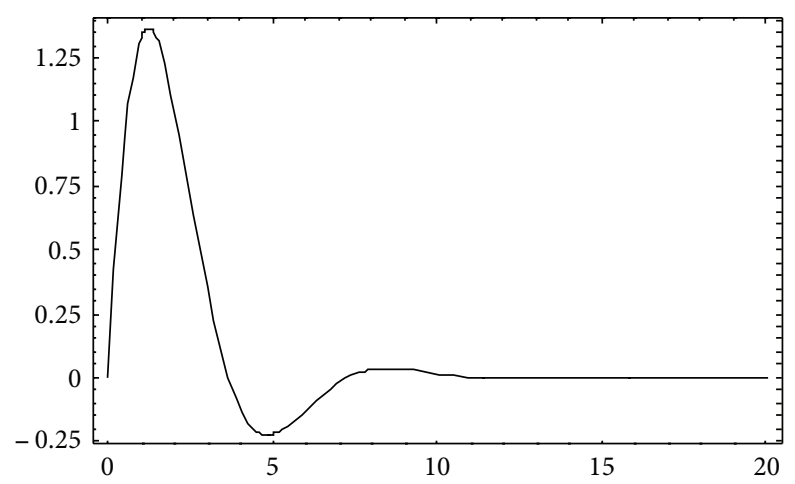

Figure 9: Time series analysis of $e_{1}(f)$.

then the eigen values of the coefficient matrix of error system (19) must be real or complex with negative real parts, and, hence, stable synchronized dynamics between systems (7) and $(8)$ is guaranteed. Let

$$
a+d=c(1+b)-a d=-E,
$$

where $E>0$ is a real number which is usually set equal to 1 . There are several ways of choosing the constant elements $a$, $b, c, d$ of matrix $D$ in order to satisfy the Lyapunov stability theory and the Routh-Hurwitz criteria (19).

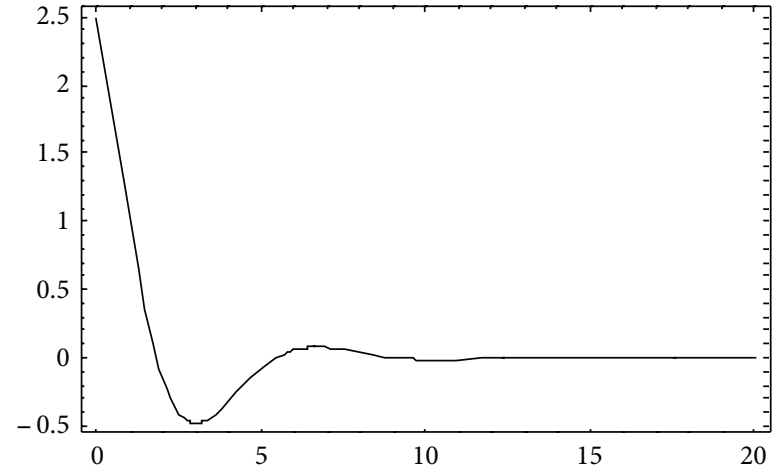

Figure 10: Time series analysis of $e_{2}(f)$.

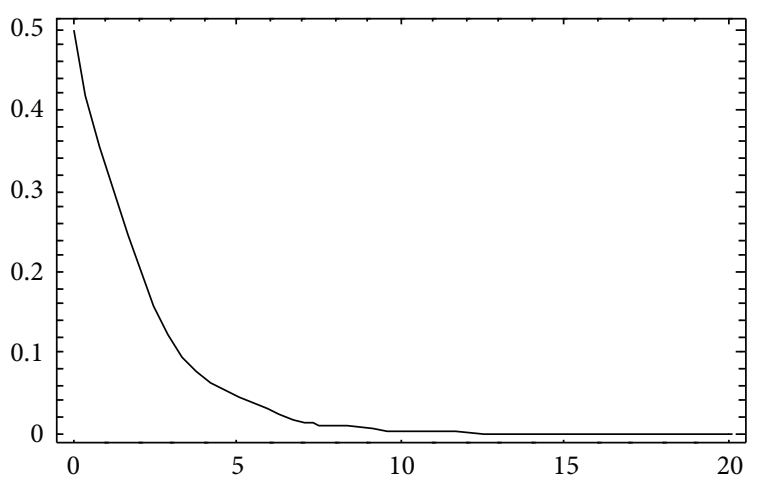

FIgURE 11: Convergence of errors in synchronization.

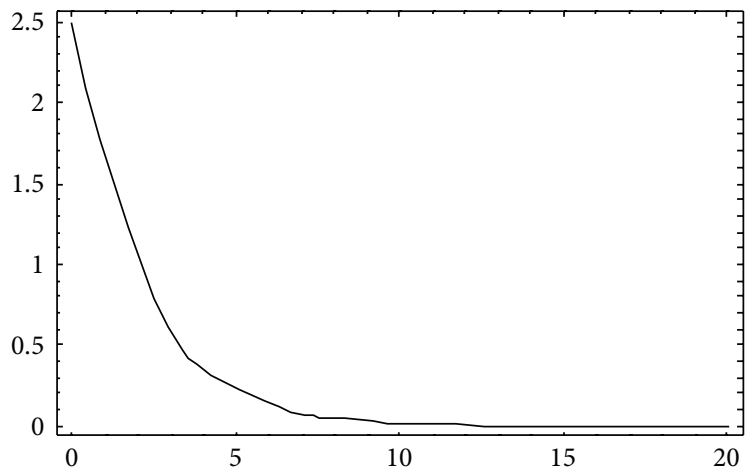

FIGURE 12: Convergence of errors in antisynchronization.

\section{Numerical Simulation for Antisynchronization}

For the constant elements of feedback matrix, choosing $a=$ $d=-0.5$ and for the parameters involved in system under investigation, $e=0.15, \omega_{0}=0.3$ with the initial conditions $\left[x_{1}(0), y_{1}(0)\right]=[0,0]$ and $\left[x_{2}(0), y_{2}(0)\right]=[1,1.5]$, we have simulated the system under consideration using mathematica. The results obtained show that the system under consideration achieved synchronization. Phase portraits, time series analysis, and error diagrams are the witness of achieving synchronization between master and slave system. 
Further, it also has been confirmed by the convergence of the synchronization quality defined by

$$
e(f)=\sqrt{e_{1}^{2}(f)+e_{2}^{2}(f)} .
$$

Figures 7-12 confirms the convergence of the synchronization quality defined by (24).

\section{Conclusion}

In this paper, we have investigated the synchronization and antisynchronization behaviour of the two identical planar oscillation of a satellite in elliptic orbit evolving from different initial conditions via the active control technique based on the Lyapunov stability theory and the Routh-Hurwitz criteria. The results were validated by numerical simulations using mathematica. For the errors in synchronization and antisynchronization behavior of the system under study, we have observed that the rate of convergence of errors is faster in antisynchronization. Figures 1-12 are proof of it.

\section{References}

[1] L. M. Pecora and T. L. Carroll, "Synchronization in chaotic systems," Physical Review Letters, vol. 64, no. 8, pp. 821-824, 1990.

[2] M. Lakshmanan and K. Murali, Chaos in Nonlinear Oscillators: Controlling and Synchronizing, World Scientist, Singapore, 1996.

[3] A. Pikovsky, M. Rosenblum, and J. Kurths, Synchronization. A Unified Approach to Nonlinear Science, Cambridge University Press, Cambridge, UK, 2001.

[4] C. M. Ticos, E. Rosa Jr., W. B. Pardo, J. A. Walkenstein, and M. Monti, "Experimental real-time phase synchronization of a paced chaotic plasma discharge," Physical Review Letters, vol. 85, no. 14, pp. 2929-2932, 2000.

[5] L. Lu, C. Zhang, and Z. A. Guo, "Synchronization between two different chaotic systems with nonlinear feedback control," Chinese Physics, vol. 16, no. 6, pp. 1603-1607, 2007.

[6] Y. Wang, Z. H. Guan, and H. O. Wang, "Feedback and adaptive control for the synchronization of Chen system via a single variable," Physics Letters, Section A, vol. 312, no. 1-2, pp. 3440, 2003.

[7] J. H. Park, "Chaos synchronization between two different chaotic dynamical systems," Chaos, Solitons and Fractals, vol. 27 , no. 2, pp. 549-554, 2006.

[8] M. Haeri and A. Emadzadeh, "Synchronizing different chaotic systems using active sliding mode control," Chaos, Solitons and Fractals, vol. 31, no. 1, pp. 119-129, 2007.

[9] E. W. Bai and K. E. Lonngren, "Synchronization of two Lorenz systems using active control," Chaos, Solitons \& Fractals, vol. 9, pp. 1555-1561, 1998.

[10] E. W. Bai, K. E. Lonngren, and J. C. Sprott, "On the synchronization of a class of electronic circuits that exhibit chaos," Chaos, Solitons \& Fractals, vol. 13, no. 7, pp. 1515-1521, 2002.

[11] H. K. Chen, "Synchronization of two different chaotic systems: a new system and each of the dynamical systems Lorenz, Chen and Lü," Chaos, Solitons \& Fractals, vol. 25, no. 5, pp. 10491056, 2005.

[12] U. E. Vincent, "Synchronization of Rikitake chaotic attractor using active control," Physics Letters, Section A, vol. 343, no. 1-3, pp. 133-138, 2005.
[13] U. E. Vincent, "Synchronization of identical and non-identical 4-D chaotic systems using active control," Chaos, Solitons \& Fractals, vol. 37, no. 4, pp. 1065-1075, 2008.

[14] A. N. Njah and U. E. Vincent, "Synchronization and antisynchronization of chaos in an extended Bonhöffer-van der Pol oscillator using active control," Journal of Sound and Vibration, vol. 319, no. 1-2, pp. 41-49, 2009.

[15] A. N. Njah, "Synchronization of forced damped pendulum via active control," Journal of the Nigerian Association of Mathematical Physics, vol. 10, pp. 143-148, 2006.

[16] A. Ucar, E. W. Bai, and K. E. Lonngren, "Synchronization of chaotic behavior in nonlinear Bloch equations," Physics Letters, Section A, vol. 314, no. 1-2, pp. 96-101, 2003.

[17] Y. Lei, W. Xu, J. Shen, and T. Fang, "Global synchronization of two parametrically excited systems using active control," Chaos, Solitons \& Fractals, vol. 28, no. 2, pp. 428-436, 2006.

[18] U. E. Vincent and A. Ucar, "Synchronization and antisynchronization of chaos in permanent magnet reluctance machine," Far East Journal of Dynamical Systems, vol. 9, pp. 211-221, 2007.

[19] U. E. Vincent and J. A. Laoye, "Synchronization, anti-synchronization and current transports in non-identical chaotic ratchets," Physica A, vol. 384, no. 2, pp. 230-240, 2007.

[20] A. Ucar, K. E. Lonngren, and E. W. Bai, "Chaos synchronization in RCL-shunted Josephson junction via active control," Chaos, Solitons \& Fractals, vol. 31, no. 1, pp. 105-111, 2007.

[21] H. Zhu and X. Zhang, "Modified projective synchronization of different hyperchaotic systems," Journal of Information and Computing Science, vol. 4, pp. 33-40, 2009.

[22] V. V. Beletskii, Motion of an Artificial Satellite about Its Center of Mass, 1966, Jerusalem: Israel Program Science Translation.

[23] V. V. Beletskii, M. L. Pivovarov, and E. L. Starostin, "Regular and chaotic motions in applied dynamics of a rigid body," Chaos, vol. 6, no. 2, pp. 155-166, 1996.

[24] R. B. Singh and V. G. Demin, "About the motion of a heavy flexible string attached to the satellite in the central field of attraction," Celestial Mechanics \& Dynamical Astronomy, vol. 6, no. 3, pp. 268-277, 1972.

[25] C. Soto-Trevino and T. J. Kaper, "Higher-order Melnikov theory for adiabatic systems," Journal of Mathematical Physics, vol. 37, no. 12, pp. 6220-6249, 1996.

[26] L. S. Wang, P. S. Krishnaprasad, and J. H. Maddocks, "Hamiltonian dynamics of a rigid body in a central gravitational field," Celestial Mechanics \& Dynamical Astronomy, vol. 50, no. 4, pp. 349-386, 1991.

[27] J. Wisdom, "Rotational dynamics of irregularly shaped natural satellites," The Astronomical Journal, vol. 94, pp. 1350-1360, 1987.

[28] J. Wisdom, S. J. Peale, and F. Mignard, "The chaotic rotation of Hyperion,” Icarus, vol. 58, no. 2, pp. 137-152, 1984.

[29] P. Goldreich and S. Peale, "Spin-orbit coupling in the solar system," The Astronomical Journal, vol. 71, pp. 425-438, 1966.

[30] A. Khan, R. Sharma, and L. M. Saha, "Chaotic motion of an ellipsoidal satellite I," The Astronomical Journal, vol. 116, no. 4, pp. 2058-2066, 1998.

[31] C. D. Murray and S. F. Dermott, Solar System Dynamics, Cambridge, UK, Cambridge University Press, 1999.

[32] H. Nijmeijer and M. Y. Mareels, "An observer looks at synchronization," IEEE Transactions on Circuits and Systems, vol. 44, no. 10, pp. 882-890, 1997.

[33] C. M. Kim, S. Rim, W. H. Kye, J. W. Ryu, and Y. J. Park, "Antisynchronization of chaotic oscillators," Physics Letters, Section A, vol. 320, no. 1, pp. 39-46, 2003. 
[34] A. A. Emadzadeh and M. Haeri, "Anti-synchronization of two different chaotic systems via active control," in Proceedings of the 4th World Enformatika Conference, (WEC '05), pp. 62-65, June 2005.

[35] C. Li and X. Liao, "Anti-synchronization of a class of coupled chaotic systems via linear feedback control," International Journal of Bifurcation \& Chaos, vol. 16, no. 4, pp. 1041-1047, 2006.

[36] S. Nakata, T. Miyata, N. Ojima, and K. Yoshikawa, "Self-synchronization in coupled salt-water oscillators," Physica D, vol. 115, no. 3-4, pp. 313-320, 1998.

[37] G. Cai and S. Zheng, "Anti-synchronization in different hyperchaotic systems," Journal of Information and Computing Science, vol. 3, pp. 181-188, 2008. 

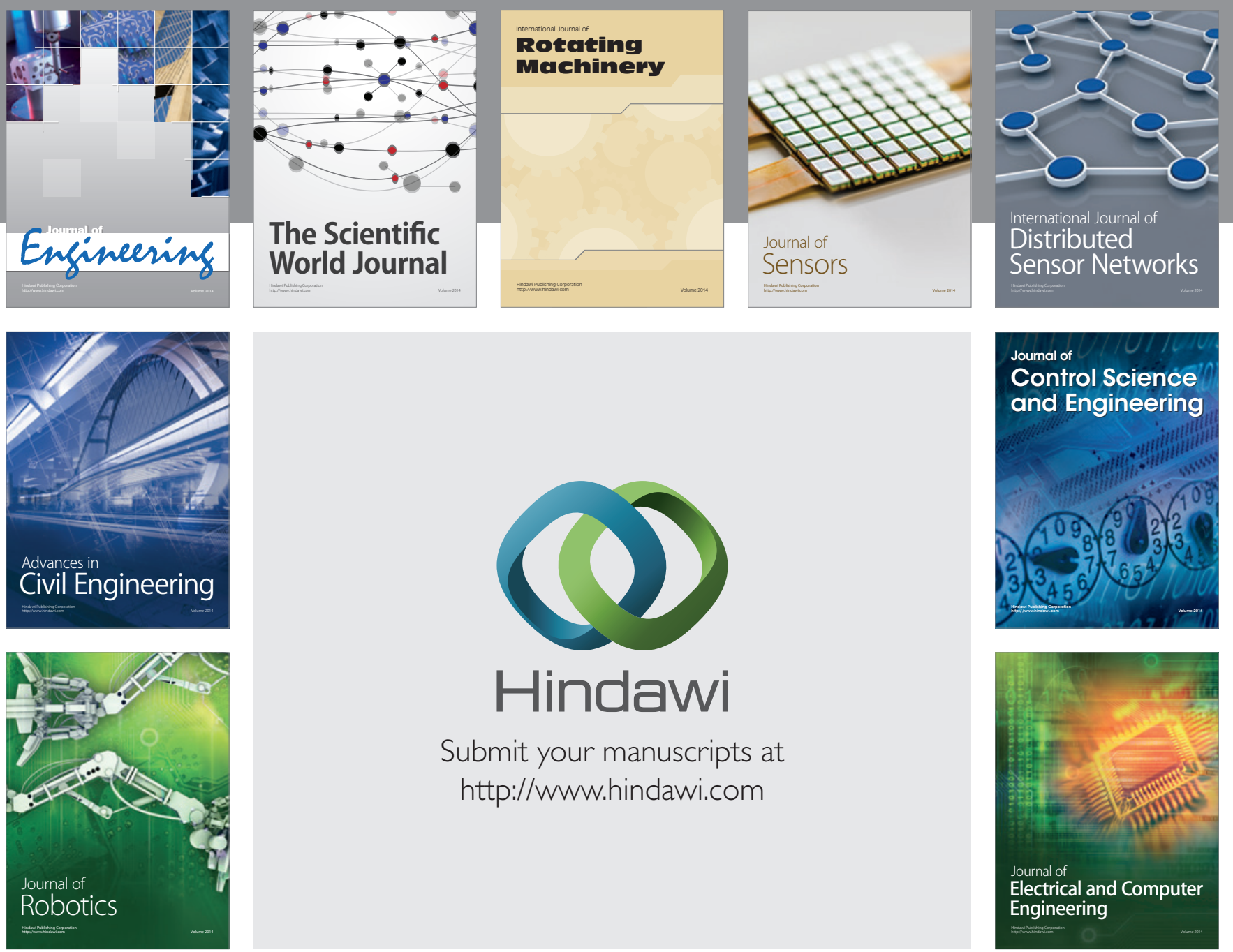

Submit your manuscripts at

http://www.hindawi.com
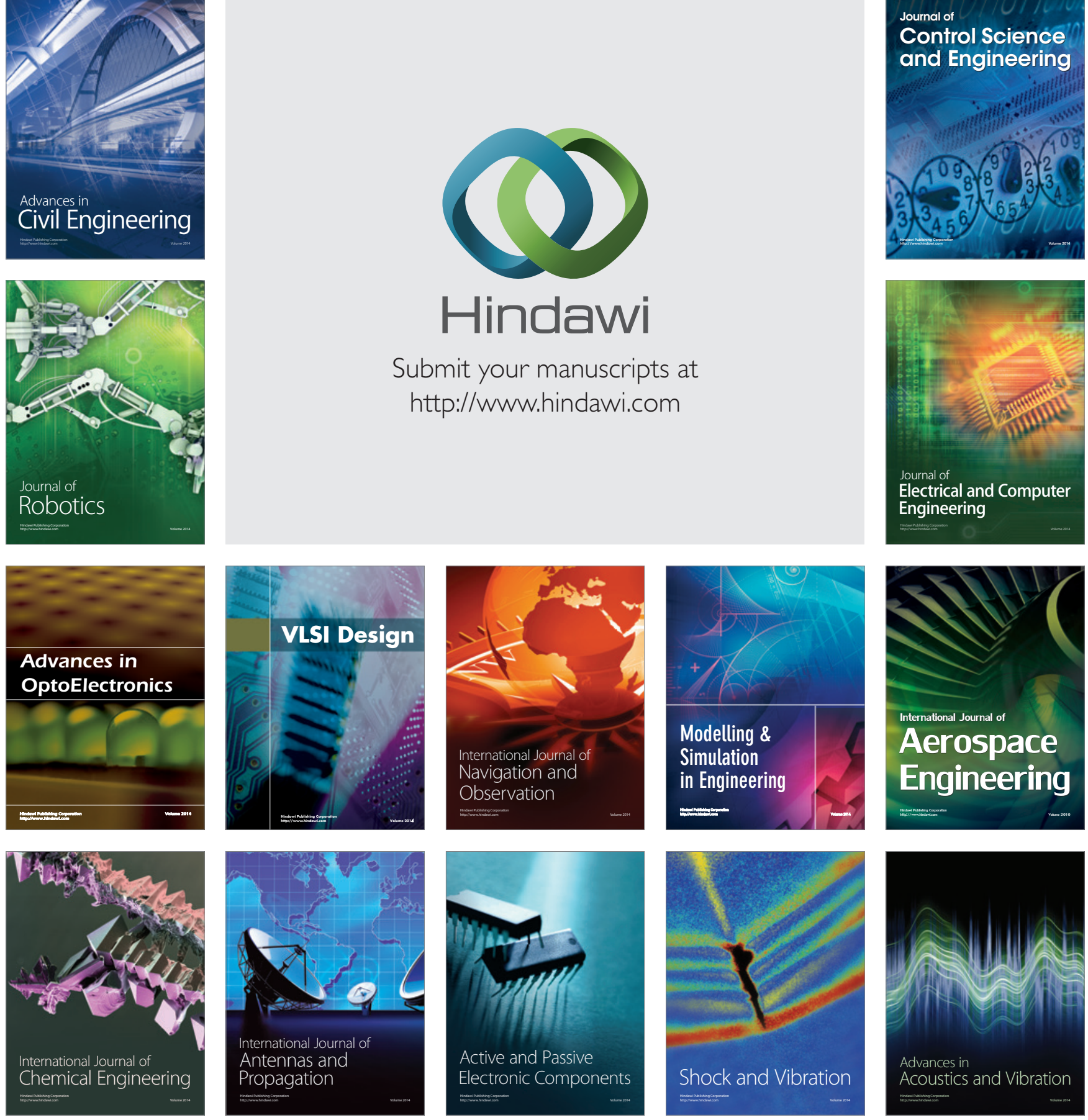